doi:10.1038/pr.2013.75

\section{(Pro)renin receptor}

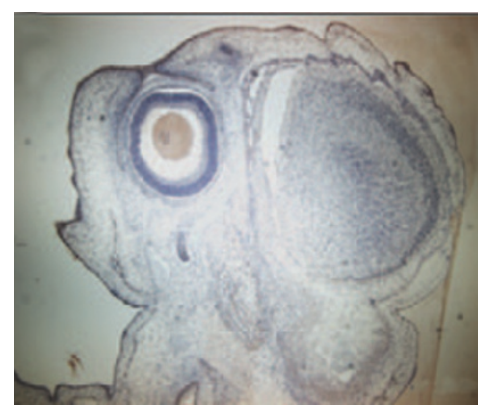

Song and colleagues examined temporal expression of the (pro) renin receptor ((P)RR) during organogenesis in mice. Investigators found that (P)RR gene and protein expression in the brain, kidney, heart, and lung is developmentally regulated in a tissue-specific manner. See page 5

\section{Hyperactive airways}

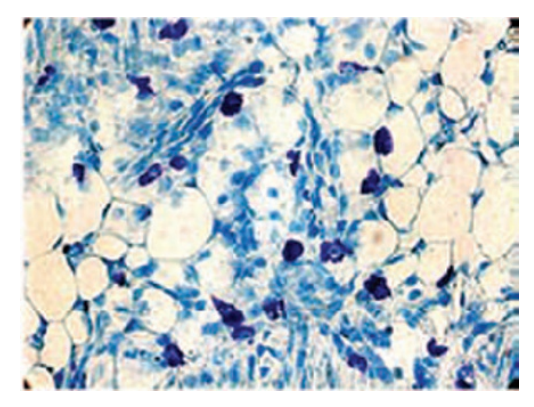

Choi and coinvestigators sought to determine whether the priming effect of intra-amniotic lipopolysaccharide (LPS) amplifies hyperoxia-induced airway hyperreactivity $(A H R)$ in rat pups. LPS was injected into the amniotic cavities of pregnant rats, and rat pups were exposed to $60 \% \mathrm{O}_{2}$ for 14 days after birth. On postnatal day 14 , it was found that LPS had indeed amplified postnatal hyperoxia-induced AHR. See page 11

\section{Hyperoxic lung injury}

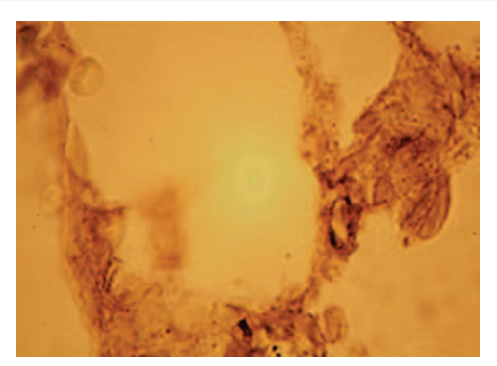

Bronchopulmonary dysplasia (BPD) is an important cause of morbidity among preterm newborns. Cetinkaya et al. evaluated the preventive effect of cytidine $5^{\prime}$-diphosphocholine (CDP choline) on hyperoxic lung injury in a neonatal rat model. Severe hyperoxia-induced lung damage was significantly ameliorated by CDP-choline treatment, suggesting that this may be a novel therapeutic option for the prevention of BPD. See page 26

\section{Imaging and brain maturation}

Natalucci and coauthors hypothesized that early amplitudeintegrated electroencephalogram (aEEG) values correlate with brain maturation and injury score assessed by conventional magnetic resonance imaging (MRI) at term-equivalent age. The results of their study show that the average aEEG total maturity

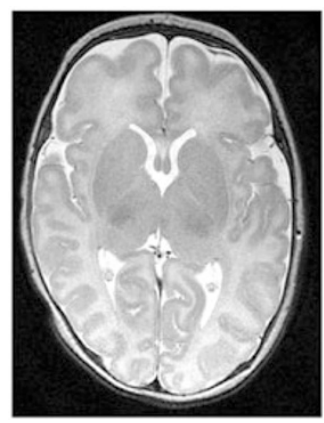

score and its cycling subscore were positively and significantly associated with total MRI maturation score. See page 68

\section{Spectroscopy and the preterm brain}

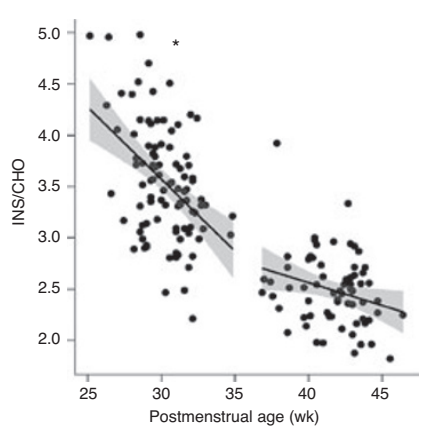

Magnetic resonance spectroscopy enables noninvasive study of brain metabolism and may thus provide useful information about brain injury. Card and colleagues identified associations of brain metabolite ratios in very preterm infants with white matter lesions and overall health status at birth. They found that both overall health status and white matter injury are reflected in metabolite ratios measured shortly after birth. See page 75 